\title{
Tumor-Infiltrating Lymphocytes in Patients Receiving Trastuzumab/Pertuzumab-Based Chemotherapy: A TRYPHAENA Substudy
}

\author{
Michail Ignatiadis*, Gert Van den Eynden*, Salgado Roberto*, Marco Fornili, Yacine Bareche, \\ Christine Desmedt, Françoise Rothé, Marion Maetens, David Venet, Esther Holgado, \\ Virginia McNally, Astrid Kiermaier, Heidi M. Savage, Timothy R. Wilson, Javier Cortes, \\ Andreas Schneeweiss, Karen Willard-Gallo, Elia Biganzoli ${ }^{\dagger}$, Christos Sotiriou ${ }^{\dagger}$
}

See the Notes section for the full list of authors' affiliations.

Correspondence to: Michail Ignatiadis, MD, PhD, Department of Medical Oncology and Breast Cancer Translational Research Laboratory, J. C. Heuson, Institut Jules Bordet, Université Libre de Bruxelles, Rue Héger-Bordet 1, 1000 Brussels, Belgium (e-mail: michail.ignatiadis@bordet.be).

*Equal contribution

†Equal contribution

\begin{abstract}
Background: There is an urgent requirement to identify biomarkers to tailor treatment in human epidermal growth factor receptor 2 (HER2)-amplified early breast cancer treated with trastuzumab/pertuzumab-based chemotherapy.

Methods: Among the 225 patients randomly assigned to trastuzumab/pertuzumab concurrently or sequentially with an anthracycline-containing regimen or concurrently with an anthracycline-free regimen in the Tryphaena trial, we determined the percentage of tumor-infiltrating lymphocytes (TILs) at baseline in 213 patients, of which 126 demonstrated a pathological complete response (pCR; ypT0/is ypNO), with 28 demonstrating event-free survival (EFS) events. We investigated associations between baseline TIL percentage and either pCR or EFS after adjusting for clinicopathological characteristics using logistic and Cox regression models, respectively. To understand TIL biology, we evaluated associations between baseline TILs and baseline tumor gene expression data ( 800 gene set by NanoString) in a subset of 173 patients. All statistical tests were two-sided.

Results: Among the patients with measurable TILs at baseline, the median level was $14.1 \%$ (interquartile range $=7.1 \%-32.4 \%$ ). After adjusting for clinicopathological characteristics, baseline percentage TIL was not associated with pCR (adjusted odds ratio $[\mathrm{aOR}]$ for every 10 -percentage unit increase in TILs $=1.12,95 \%$ confidence interval $[\mathrm{CI}]=0.95$ to $1.31, \mathrm{P}=.17)$. At a median follow-up of 4.7 years, for every increase in baseline TILs of $10 \%$, there was a $25 \%$ reduction in the hazard for an EFS event $(\mathrm{aOR}=0.75,95 \% \mathrm{CI}=0.56$ to $1.00, \mathrm{P}=.05)$ after adjusting for baseline clinicopathological characteristics and $\mathrm{pCR}$. Additionally, genes associated with epithelial-mesenchymal transition, angiogenesis, and T-cell inhibition such as SNAIL1, ZEB1, NOTCH3, and B7-H3 were statistically significantly inversely correlated with percentage TIL.

Conclusions: Baseline TIL percentage provides independent prognostic information in patients treated with trastuzumab/ pertuzumab-based neoadjuvant chemotherapy. However, further validation is required.
\end{abstract}


The percentage of stromal tumor-infiltrating lymphocytes (TILs) was associated with an increased pathological complete response $(\mathrm{pCR})$ rate in patients with human epidermal growth factor receptor 2 (HER2)-amplified breast cancer receiving either neoadjuvant anthracycline and taxane-based chemotherapy alone in the GeparDuo and GeparTrio trials (1) or weekly paclitaxel with trastuzumab or lapatinib or both in the NeoALTTO trial (2). Similarly, high expression of immune-related genes/ gene signatures was associated with increased PCR rates in patients who received neoadjuvant anthracycline and taxanebased chemotherapy alone (3) or weekly paclitaxel in combination with trastuzumab and lapatinib (4). In the Neosphere trial (randomizing patients between trastuzumab plus docetaxel vs trastuzumab/pertuzumab plus docetaxel vs trastuzumab/pertuzumab without chemotherapy vs pertuzumab plus docetaxel), TIL percentage as a continuous variable was not associated with PCR (5).

However, TIL percentage as a continuous variable $(10 \%$ increase in level of TILs) was associated with improved distant recurrence-free survival in the adjuvant trastuzumab arm of the Finher Trial (6). Furthermore, TIL percentage as a continuous variable was associated with improved event-free survival (EFS) in the NeoALTTO trial (2). However, in the pivotal N9831 Adjuvant Trial, TIL percentage as a continuous variable $(10 \%$ increase in level of TILs) was not associated with a recurrence-free interval in the trastuzumab arm (7).

No data are available for the prognostic associations of TILs with PCR and EFS in patients with early breast cancer receiving standard neoadjuvant chemotherapy (anthracycline/taxane or carboplatine/taxane) with dual HER2 blockade with trastuzumab and pertuzumab. We aimed to study these associations in the context of the TRYPHAENA neoadjuvant study (8).

\section{Methods}

\section{TRYPHAENA Study Design}

The TRYPHAENA study (NCT00976989) design and results have been previously reported (8) and are described in the Supplementary Methods (available online). Written informed consent was obtained from each participant. Approval for the protocol and for any modifications was obtained from independent ethics committees (committees are listed in the Supplementary Methods, available online).

\section{Stromal Tumor-Infiltrating Lymphocyte Evaluation}

The percentages of stromal coverage by TILs (hereafter referred to as TIL percentage) before (at diagnosis) and after (for patients with residual invasive breast cancer) dual HER2 blockade were evaluated in hematoxylin and eosin (H\&E) slides from diagnostic biopsies and surgical samples from patients enrolled in the TRYPHAENA study. TIL percentage at surgery was evaluated in patients with residual invasive breast cancer. In the analyses, the geometric means of the percentage of TILs present were determined by two independent pathologists (9); the agreement between the two pathologists was evaluated as explained in the Supplementary Methods (available online). TIL percentage at diagnosis was assessed according to the 2014 guidelines developed by the International Immuno-Oncology Biomarker Working Group (10). TIL percentage for patients with residual invasive disease was scored according to guidelines developed by the International Immuno-Oncology Biomarker Working
Group (11). In cases of pCR or if no tumor bed could be identified, TIL assessment was not performed.

\section{Gene Expression Using the NanoString nCounter Assay}

Sample processing and RNA extraction for gene expression profiling are described in the Supplementary Methods (available online). Gene expression data from the NanoString nCounter assay (previously reported custom designed 800-gene panel tailored for breast cancer [12]) were analyzed after quality control filtering and normalization using the $\mathrm{R}$ package NanoStringQCPro (version 0.99.8.3). As recommended, the mean of the positive probes and the housekeeping genes were used in order to account for differences in sample input. Moreover, negative control probes were used for background correction, and samples that did not pass quality control were removed. Raw and normalized NanoString data have been deposited in the Gene Expression Omnibus database under accession number GSE109710.

We calculated the expression of the ERBB2 and ESR1, genes that have been shown to be associated with PCR after neoadjuvant chemotherapy and anti-HER2 agents $(4,13)$, and the expression of well-known genes associated with CD8+ T-cell cytolytic activity: granzyme A and B (GZMA and GZMB) and perforin 1 (PRF1), with T-regulatory cells: forkhead box P3 (FOXP3), with immune checkpoint inhibition: cytotoxic T-lymphocyte-associated protein 4 (CTLA4), programmed death-ligand 1 (PDL1) and programmed cell death protein 1 (PD1), with T-helper phenotype 1: interferon-gamma (IFNG) and 2: interleukin 4 (IL4). Moreover, we computed three immune-gene signatures, a STAT1-related gene signature (immune_STAT1) (14), a signature related to the presence of tertiary lymphoid structures (immune_TLS) (15), and an immune-gene signature associated with benefit from adjuvant trastuzumab in the North Central Cancer Treatment Group N9831 adjuvant trastuzumab trial (immune_trastuzumab) (16). Details about single gene/signature scores calculations and PAM50 subtype definition are provided in the Supplementary Methods (available online).

\section{Statistical Analyses}

The association between TIL percentage, at baseline and at residual disease or surgery, with clinicopathologic variables was evaluated using linear regression. Clinicopathological variables included age ( $<50$ and $\geq 50$ years), histology grade (III vs I/II), estrogen receptor (ER) status (positive vs negative), clinical stage (III vs II), and chemotherapy (anthracyclines vs nonanthracyclines). The dependence of PCR, defined as absence of invasive tumor in the breast and lymph nodes (ypT0/is ypNO), on TIL percentage at baseline and clinicopathologic variables was assessed using logistic regression models.

The presence of nonlinear effects of TIL percentage at baseline on PCR probability on the logit scale, modeled by restricted cubic splines, was evaluated by Wald test. pCR probabilities according to TIL quartiles at baseline were also estimated, with $95 \%$ confidence intervals (CIs) computed by the Wilson method. EFS was defined as the time from the date of surgery to the first documentation of disease progression or death. Any evidence of contralateral disease in situ was not considered disease progression. EFS was described separately in patients who achieved a pCR from those who did not. Patients who were withdrawn from the study without documented disease progression were censored at the date of the last assessment when the patient 


\begin{tabular}{|c|c|c|c|c|}
\hline Variable & $\begin{array}{l}\text { All patients } \\
\text { No. }(\%)\end{array}$ & $\begin{array}{c}\text { Patients with evaluable } \\
\text { TILs* at baseline } \\
\text { No. (\%) }\end{array}$ & $\begin{array}{c}\text { Patients with evaluable } \\
\text { TILs at surgery and } \\
\text { no pCR } \\
\text { No. (\%) }\end{array}$ & $\begin{array}{c}\text { Patients with available } \\
\text { gene expression data at } \\
\text { baseline } \\
\text { No. (\%) }\end{array}$ \\
\hline Total & $225(100.0)$ & $213(100.0)$ & $64(100.0)$ & $173(100.0)$ \\
\hline \multicolumn{5}{|l|}{ Age, y } \\
\hline$<50$ & $112(49.8)$ & $108(50.7)$ & $41(64.1)$ & 89 (51.4) \\
\hline$\geq 50$ & $113(50.2)$ & $105(49.3)$ & $23(35.9)$ & $84(48.6)$ \\
\hline \multicolumn{5}{|l|}{ Histology grade } \\
\hline I/II & $101(56.4)$ & $95(55.2)$ & $36(66.7)$ & $74(52.9)$ \\
\hline III & $78(43.6)$ & $77(44.8)$ & $18(33.3)$ & $66(47.1)$ \\
\hline Unknown & 46 & 41 & 10 & 33 \\
\hline \multicolumn{5}{|l|}{ Estrogen receptor* } \\
\hline Negative & $118(52.7)$ & $112(52.6)$ & $20(31.7)$ & $94(54.3)$ \\
\hline Positive & $106(47.3)$ & $101(47.4)$ & $43(68.3)$ & $79(45.7)$ \\
\hline Unknown & 1 & - & 1 & - \\
\hline \multicolumn{5}{|l|}{ Clinical stage $†$} \\
\hline II & $115(51.6)$ & $110(52.1)$ & $27(42.2)$ & $92(53.5)$ \\
\hline III & $108(48.4)$ & $101(47.9)$ & $37(57.8)$ & $80(46.5)$ \\
\hline Unknown & 2 & 2 & - & 1 \\
\hline \multicolumn{5}{|l|}{ Chemotherapy } \\
\hline Non-anthracycline-based & 77 (34.2) & $72(33.8)$ & $17(26.6)$ & $59(34.1)$ \\
\hline Anthracycline-based & $148(65.8)$ & $141(66.2)$ & $47(73.4)$ & $114(65.9)$ \\
\hline \multicolumn{5}{|l|}{ pCR (ypTo/is ypNo) } \\
\hline No & $94(41.8)$ & $87(40.8)$ & $64(100.0)$ & $70(40.5)$ \\
\hline Yes & $131(58.2)$ & $126(59.2)$ & - & $103(59.5)$ \\
\hline EFS, No. of events & 29 & 28 & 14 & 26 \\
\hline
\end{tabular}

${ }^{*}$ Estrogen receptor status evaluated using immunohistochemistry. EFS = event-free survival; pCR = pathological complete response; TILs = tumor-infiltrating lymphocytes.

†Clinical stage assessed using the 7th edition of the American Joint Committee on Cancer Staging.

was known to be disease-free. Median follow-up was obtained by the reverse Kaplan-Meier method. Association of EFS with TIL percentage and clinicopathological variables was examined using the Cox regression model. All tests of statistical significance were two-sided, and a $P$ value of less than .05 was considered statistically significant.

Statistical analysis of gene expression data generated using the NanoString nCounter Assay is described in the Supplementary Methods (available online). This study has been reported according to the Reporting Recommendations for Tumor Marker Prognostic Studies (REMARK) criteria (17).

\section{Results}

\section{Patient Characteristics}

The patient characteristics for the entire TRYPHAENA cohort, the subgroups of patients with evaluable TILs at baseline and at the point of surgery, and available gene expression data at baseline are summarized in Table 1. TIL status was successfully evaluated in 213 (94.7\%) of 225 patients at baseline and in 64 (73.6\%) of 87 patients with invasive residual disease at the point of surgery. The reasons behind unsuccessful evaluation are presented in the CONSORT diagram for TILs (Supplementary Figure 1 , available online). The main reason for missing results at the point of surgery was either because patients had PCR or because the pathologists were not able to identify the tumor bed in available H\&E slides ( $\mathrm{n}=140$ patients) (Supplementary Figure 1, available online). Agreement between the two pathologists was good (Supplementary Figure 2, available online). For Log TILs at baseline, the concordance correlation coefficient was $0.62(95 \% \mathrm{CI}=0.55$ to 0.68$)$, while at surgery it was $0.66(95 \% \mathrm{CI}=$ 0.59 to 0.73 ).

The expression of immune-related single genes and gene signatures at baseline in tumor samples was successfully evaluated using NanoString technology in 173 of 225 (76.9\%) patients. A lack of sample was the main reason for missing data $(n=41)$, with additional reasons described in the CONSORT diagram for NanoString gene expression data (Supplementary Figure 3, available online).

\section{TIL Percentage at Baseline and Baseline Clinicopathological Characteristics}

We first tested whether there was any association between TIL percentage and clinicopathological characteristics at baseline. No evidence of association was found between TIL percentage and age, ER status, or type of neoadjuvant chemotherapy (anthracycline-based vs other) (Supplementary Table 1, available online). However, there was an association between an increased TIL percentage at baseline and histological grade III tumors $(P=.03)$.

\section{TIL Percentage at Baseline and PCR}

No evidence for a nonlinear relationship between $\mathrm{pCR}$ probability on the logit scale and TIL percentage at baseline was found. Figure 1 and Supplementary Figure 4 (available online) depict pCR rates according to TIL percentage at baseline assessed as a categorical variable using quartiles and a scatterplot of pCR 


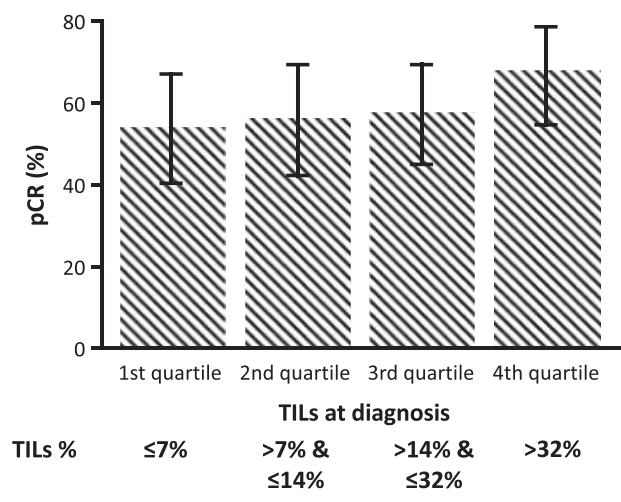

\begin{tabular}{lccc}
\hline Quartile & $\begin{array}{c}\text { All } \\
\mathbf{n ~ ( \% ) ~}\end{array}$ & $\begin{array}{c}\text { pCR } \\
\mathbf{n}(\%)\end{array}$ & $\begin{array}{c}\text { No pCR } \\
\mathbf{n}(\%)\end{array}$ \\
\hline $\mathbf{1}^{\text {st }}$ & $50(100)$ & $27(54.0)$ & $23(46.0)$ \\
$\mathbf{2}^{\text {nd }}$ & $48(100)$ & $27(56.2)$ & $21(43.8)$ \\
$\mathbf{3}^{\text {rd }}$ & $59(100)$ & $34(57.6)$ & $25(42.4)$ \\
$\mathbf{4}^{\text {th }}$ & $56(100)$ & $38(67.9)$ & $18(32.1)$ \\
\hline
\end{tabular}

$95 \%$ confidence intervals, computed according to the Wilson method. pCR = pathological complete response; TIL = tumor-infiltrating lymphocyte.

Table 2. Univariate associations between variables and pathologic complete response and event-free survival

\begin{tabular}{|c|c|c|c|c|c|c|}
\hline \multirow[b]{2}{*}{ Variable } & \multicolumn{3}{|c|}{ Univariate for $\mathrm{pCR}^{*}$} & \multicolumn{3}{|c|}{ Univariate for EFS } \\
\hline & $\begin{array}{l}\text { No. of patients } \\
\text { (pCR event) }\end{array}$ & OR $(95 \%$ CI $)$ & $P^{*}$ & $\begin{array}{l}\text { No. of patients } \\
\text { (EFS event) }\end{array}$ & HR $(95 \%$ CI) & $\mathrm{P} \dagger$ \\
\hline Age: $\geq 50$ vs $<50 y$ & $225(131)$ & 1.47 (0.86 to 2.50$)$ & .16 & $208(29)$ & 1.54 (0.74 to 3.23$)$ & .25 \\
\hline Histology grade: III vs I/II & $179(101)$ & 1.59 (0.87 to 2.91$)$ & .13 & $166(26)$ & 0.71 (0.31 to 1.58$)$ & .40 \\
\hline Estrogen receptor: positive vs negative & $224(131)$ & $0.32(0.19$ to 0.56$)$ & $<.001$ & $207(29)$ & 0.94 (0.45 to 1.94$)$ & .86 \\
\hline Clinical stage: III vs II & $223(130)$ & $0.64(0.38$ to 1.10$)$ & .11 & $207(28)$ & 2.45 (1.11 to 5.42$)$ & .03 \\
\hline Chemotherapy: anthracycline-based vs not & $225(131)$ & $0.71(0.40$ to 1.25$)$ & .24 & $208(29)$ & 0.83 (0.39 to 1.76$)$ & .63 \\
\hline Baseline TILs (10-unit increase) & $213(126)$ & 1.15 (0.99 to 1.32$)$ & .06 & $198(28)$ & 0.80 (0.63 to 1.02$)$ & .08 \\
\hline TILs at surgery $\ddagger$ (10-unit increase) & - & - & - & $63(14)$ & $1.05(0.73$ to 1.50$)$ & .80 \\
\hline pCR: yes vs no & - & - & - & $208(29)$ & 0.35 (0.16 to 0.73$)$ & .005 \\
\hline
\end{tabular}

${ }^{*} \mathrm{P}$ values based on two-sided Wald tests from univariate logistic regression models. $\mathrm{CI}=$ confidence interval; $\mathrm{EFS}=$ event-free survival; HR $=$ hazard ratio; OR= odds ratio; $\mathrm{pCR}=$ pathologic complete response; TILs = tumor-infiltrating lymphocytes. $\dagger P$ values based on two-sided Wald tests from univariate Cox regression models. $\ddagger$ Patients with no pCR.

rates across several TIL percentage cutoffs, respectively. In a univariate analysis, TIL percentage at baseline was not significantly associated with pCR (odds ratio $[\mathrm{OR}]=1.15,95 \% \mathrm{CI}=0.99$ to $1.32, P=.06$ ) (Table 2). In this study, among other baseline clinicopathological characteristics, only ER status was significantly associated with PCR, with ER-positive tumors having a lower probability for PCR (OR $=0.32,95 \% \mathrm{CI}=0.19$ to $0.56, \mathrm{P}<$ .001) (Table 2). In a multivariable model including baseline clinicopathological characteristics and percentage TILs, TILs were not significantly associated with pCR (OR for every 10-percentage unit increase in TILs $=1.12,95 \% \mathrm{CI}=0.95$ to $1.31, \mathrm{P}=.17$ ) (Supplementary Table 2, available online). Only ER-positive and higher-clinical stage tumors were independently associated with a lower probability of $\mathrm{PCR}$.

\section{TIL Percentage at Baseline and EFS}

Next, we investigated whether there was association between TILs at baseline and EFS. After a median follow-up of 4.7 years, for every $10 \%$ increase in TILs at baseline, EFS increased, but this change was not statistically significant, with a hazard ratio (HR) of 0.80 (95\% CI $=0.63$ to $1.02, P=.08$ ) (Table 2). When we examined associations between baseline clinicopathological characteristics and EFS, a higher clinical stage was associated with a shorter EFS (HR $=2.45,95 \% \mathrm{CI}=1.11$ to $5.42, P=.03$ ), whereas the presence of $\mathrm{pCR}$ was associated with longer $\mathrm{EFS}(\mathrm{HR}=0.35$,
$95 \% \mathrm{CI}=0.16$ to $0.73, \mathrm{P}=.005$ ) (Table 2). In a multivariable model including baseline clinicopathological characteristics, baseline TIL percentage, and $\mathrm{pCR}$, only $\mathrm{PCR}$ and baseline TIL percentage were independently associated with EFS (Table 3). In this model, for every 10-percentage unit increase in baseline TILs, there was a $25 \%$ reduction in the hazard for the event (adjusted $\mathrm{HR}=0.75$, $95 \% \mathrm{CI}=0.56$ to $1.00, P=.05)$. Interestingly, when we examined the EFS of patients according to the presence of pCR (yes vs no) and the level of percentage TIL (high vs low based on the median TIL value of $14.1 \%$ [interquartile range $=7.1 \%-32.4 \%$ ] in this series), we found that patients with high baseline percentage TIL and pCR had the best prognosis compared with the other groups (Figure 2). No evidence of interaction between $\mathrm{PCR}$ and baseline TIL percentage, dichotomized with cutoff of $14 \%$, was observed $(P=.10)$.

\section{TIL Percentage at the Point of Surgery}

TIL percentage at the point of surgery was evaluated in 64 of 87 patients with invasive residual disease (Supplementary Figure 1 , available online). TILs at surgery were not significantly associated with baseline clinicopathological characteristics (Supplementary Table 3, available online), or with EFS (HR = $1.05,95 \% \mathrm{CI}=0.73$ to $1.50, P=.80$ ) (Table 2), although the small number of patients and events does not allow definitive conclusions to be drawn. 
Table 3. Multivariable Cox regression (No. of patients included in the model $=159$, No. of patients with EFS event $=24$ )

\begin{tabular}{lcl}
\hline Variable & HR (95\% CI) & P* \\
\hline Age: $\geq 50$ vs $<50$ y & $1.37(0.60$ to 3.13$)$ & .46 \\
Histology grade: III vs I/II & $1.21(0.47$ to 3.07$)$ & .70 \\
Estrogen receptor: positive vs negative & $0.72(0.30$ to 1.73$)$ & .46 \\
Clinical stage: III vs II & $1.89(0.79$ to 4.51$)$ & .15 \\
Chemotherapy: anthracycline- & $0.52(0.22$ to 1.22$)$ & .13 \\
$\quad$ based vs not & $0.22(0.08$ to 0.61$)$ & .004 \\
pCR: yes vs no & $0.75(0.56$ to 1.00$)$ & .05 \\
Baseline TILs (10-unit increase) & &
\end{tabular}

${ }^{*} \mathrm{P}$ values based on two-sided Wald tests from a multivariable Cox regression model including age, histology grade, estrogen receptor status, clinical stage, chemotherapy, pCR, and baseline TILs. CI= confidence interval; $\mathrm{EFS}=$ event-free survival; $\mathrm{HR}=$ hazard ratio; $\mathrm{OR}=$ odds ratio; $\mathrm{pCR}=$ pathologic complete response; TILs = tumor-infiltrating lymphocytes

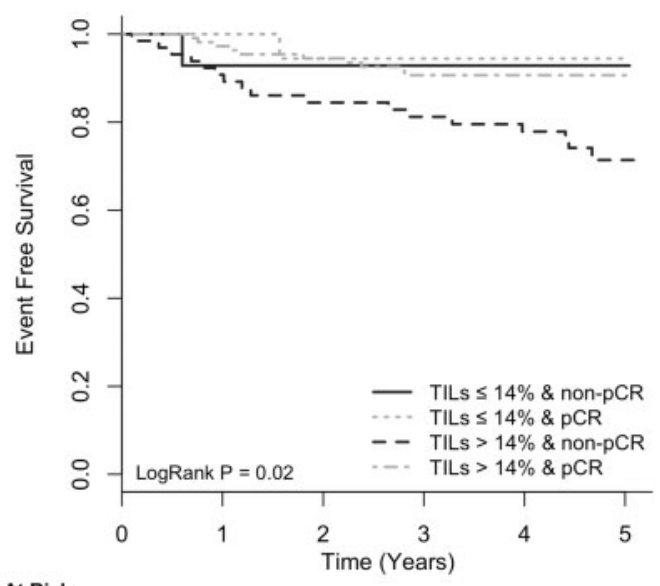

$\begin{array}{rrrrrrr}\text { No. At Risk } & & & & & & \\ \text { TILs } \leq 14 \% \text { \& non-pCR } & 15 & 13 & 12 & 10 & 10 & 1 \\ \text { TILs } \leq 14 \% \text { \& pCR } & 18 & 18 & 15 & 15 & 14 & 0 \\ \text { TILs > } 14 \% \text { \& non-pCR } & 65 & 58 & 52 & 49 & 46 & 3 \\ \text { TILs }>14 \% \text { \& pCR } & 110 & 106 & 101 & 94 & 90 & 1\end{array}$

Figure 2. Kaplan-Meier event-free survival curves of patients according to pathological complete response (pCR) status (yes vs no) and tumor-infiltrating lymphocytes (TILs) at baseline ( $>14 \%$ vs $\leq 14 \%$ ). Patients were divided into four groups: TILs $>14 \%$ and pCR, TILs $>14 \%$ and non-pCR, TILs $\leq 14 \%$ and pCR, TILs $\leq 14 \%$ and non-pCR. $\mathrm{pCR}=$ pathological complete response; $\mathrm{TIL}=$ tumor-infiltrating lymphocyte.

\section{Association Between TIL Percentage at Baseline and Gene Expression (NanoString) in Baseline Tumor Samples}

In the 173 patients for whom baseline tumor gene expression data were available, we found that TIL percentage at baseline strongly correlated with most immune genes and gene signatures, but not with ESR1 or ERBB2 (Figure 3). No association was found between TIL percentage and the PAM50 intrinsic subtype (Kruskal-Wallis $P=.48$ ) within HER2 breast cancer patients (Supplementary Figure 5, available online). To derive further insights into the tumor biology associated with an immune response as measured by TIL percentage, we evaluated associations between TIL percentage at baseline and more than 800 cancer-related genes (Figure 4A). Interestingly, gene ontology analysis showed that TIL percentage was statistically significantly inversely correlated with genes associated with epithelial mesenchymal transition (EMT), angiogenesis, and T-cell inhibition (Figure 4B), such as SNAIL1, ZEB1, NOTCH3, and B7-H3 (Figure 4C).

\section{Gene Expression (NanoString) in Baseline Tumor Samples and pCR}

Next, we investigated the association between PCR and the baseline expression of the following: single genes (ESR1, ERBB2), the PAM50 subtype (HER2-enriched subtype vs others), three immune-related gene signatures, and single immune-related genes (GZMA and GZMB, PRF1, FOXP3, CTLA4, PDL1, PD1, IL4, IFNG). After correction for multiple testing, increased $\mathrm{pCR}$ rates were observed in patients with a HER2-enriched subtype, higher expression of ERBB2, the immune_TLS and immune_trastuzumab gene signatures, and GZMA and GZMB, PRF1, PD1 and IFNG in a univariate analysis (Supplementary Figure 6, available online). Following multivariable analysis (after adjustment for baseline clinicopathological characteristics and baseline TILs) and correction for multiple testing, increased $\mathrm{pCR}$ rates were observed in patients with a higher expression of ERBB2, immune_TLS and immune_trastuzumab gene signatures, and GZMA and GZMB, PRF1 and IFNG (Figure 5). When we examined the distribution of $\mathrm{PCR}$ rates across PAM50 subtypes (Supplementary Figure 7, available online), we observed the highest response (57 out of 82 patients or 69.5\%) for the HER2enriched subtype $(P=.02)$.

\section{Gene Expression (NanoString) in Baseline Tumor Samples and EFS}

There was no association between EFS and the expression of any of the immune genes/gene signatures tested (Supplementary Figure 8, available online). In a multivariable analysis including baseline clinicopathological characteristics, PAM50 subtype, pCR, and baseline TIL percentage, pCR remained statistically significantly associated with EFS $(P=.02)$ but not with TIL percentage $(P=.06)$ (Supplementary Table 4 , available online).

\section{Discussion}

To our knowledge, this is the first study to evaluate associations between TIL percentage at baseline, PCR, and EFS following neoadjuvant chemotherapy and dual HER2 blockade with trastuzumab and pertuzumab in HER2-amplified early breast cancer. We provide for the first time evidence that TIL percentage at baseline and PCR provide independent prognostic information for EFS in patients treated with trastuzumab/pertuzumab-based neoadjuvant treatment. For every $10 \%$ increase in the level of baseline TILs, there was a $25 \%$ reduction in the occurrence of EFS events. These results need further validation in an independent patient cohort. Patients with a high baseline TIL percentage $(\geq 14 \%)$ and pathological complete response had an excellent prognosis following neoadjuvant chemotherapy and dual HER2 blockade with trastuzumab and pertuzumab. Similar results to this association between baseline TIL percentage, pCR, and EFS for the trastuzumab and lapatinib combination have been reported in the NeoALTTO trial (2).

The results of our study may have clinical implications for tailoring treatment in patients with HER2-amplified early breast cancer. For example, in 2013, pertuzumab was approved in 


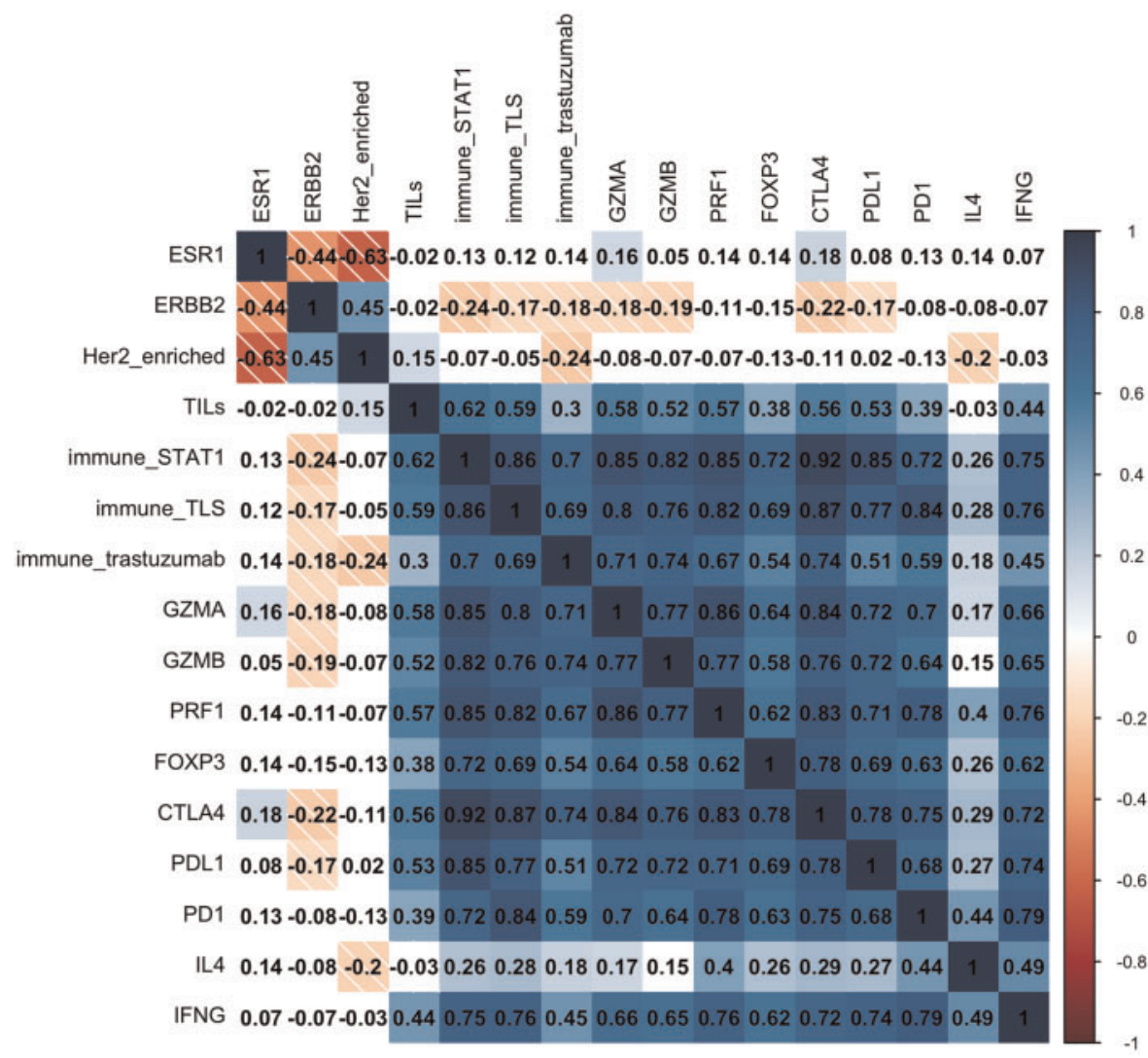

Figure 3. Heat map reflecting the hierarchical clustering of pairwise correlation between baseline tumor-infiltrating lymphocytes and baseline immune-related single genes/gene signatures in 173 patients. Cells are colored according to Spearman's correlation coefficient values, with blue indicating a positive correlation and red indicating a negative correlation.

combination with trastuzumab and docetaxel as a neoadjuvant treatment in patients with HER2-amplified breast cancer (18). Recently, the Aphinity trial demonstrated that pertuzumab, when added to trastuzumab as an adjuvant treatment, improved invasive disease-free survival by $1 \%$ (19). Due to the marginal benefit of the Aphinity trial, there is an urgent need to identify the patient subgroup that can derive most benefit from the addition of pertuzumab. For Aphinity, the subgroups predefined by clinic-pathological factors that derived the highest benefit were patients with node-positive disease and/or hormone receptor-negative disease (19); the latter was also seen in the Tryphaena study. If the results of our trial on the association between TIL percentage, PCR, and EFS are further confirmed in other studies or patient cohorts that have received neoadjuvant pertuzumab-based treatment, one might consider adding pertuzumab in high-risk HER2-positive patients as part of neoadjuvant treatment, as in the Tryphaena trial. Thus, in patients with a high baseline level of TIL percentage that achieve PCR after neoadjuvant chemotherapy and dual HER2 blockade with trastuzumab and pertuzumab, trastuzumab alone as adjuvant treatment might be the appropriate treatment due to the very good prognosis for this group. In the other patient subgroups, further clinical research is needed to identify optimal approaches that might further improve outcome (eg, evaluate the role of immunotherapy in this setting).

In our study, there was no association between TIL percentage at the point of surgery where residual invasive disease was present and EFS. However, our study was largely underpowered to detect such associations. In patients with triple-negative breast cancer (TNBC) receiving neoadjuvant chemotherapy, the presence of high levels of TILs $(>60 \%)$ in residual invasive disease was associated with improved EFS compared with patients with low levels of TILs (20). More studies with adequate sample size are needed to evaluate whether such an association exists in HER2-positive breast cancer. Moreover, it is not known whether patients with TILs at residual invasive disease might benefit from immune checkpoint inhibitors. TILs have been associated with a response to the immune checkpoint inhibitor pembrolizumab in metastatic TNBC (21). It has also been suggested that the mutational load (22), the number of neoantigens (23), and neoantigen heterogeneity (clonal vs subclonal neoantigens) (24) are associated with response to immune checkpoint inhibitors. Increased clonal neoantigen burden has been associated with increased benefit from immune checkpoint inhibitors in lung adenocarcinoma (24). Thus, the information on the presence of clonal neoantigens but also a more detailed characterization of the immune landscape $(25,26)$ of residual disease following neoadjuvant chemotherapy and dual HER2 blockade might provide more insight into the subgroup of patients that could benefit from immunotherapeutic approaches in this setting.

Additionally, we observed higher pCR rates for patients in the HER2-enriched subtype compared with other subtypes and for patients with higher ERBB2 mRNA levels, as has been observed in the CALGB 40601 (13) and NeoALTTO (4) studies.

Moreover, we demonstrated that after adjustment for baseline clinicopathological characteristics and baseline TIL 


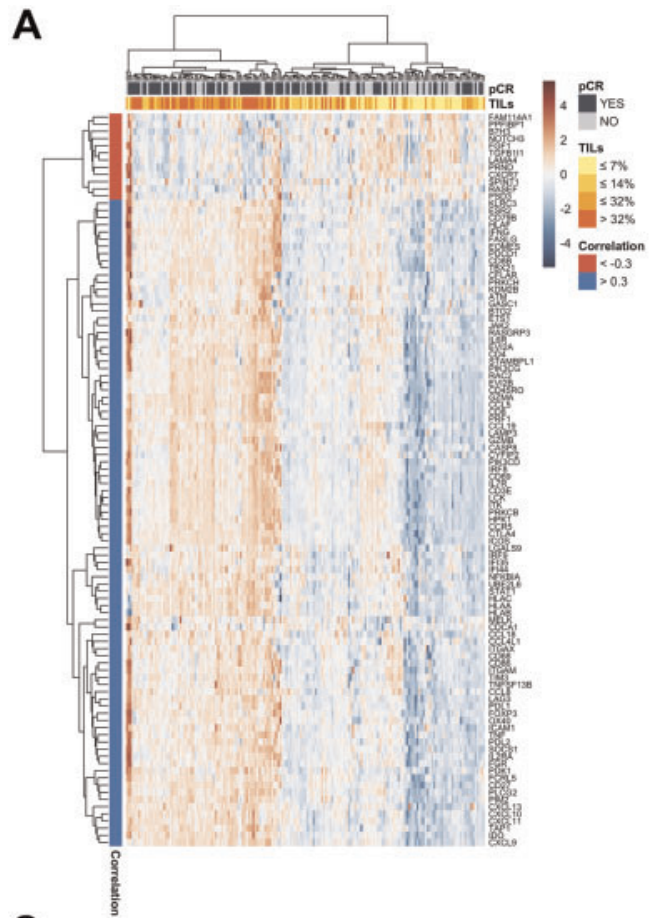

B
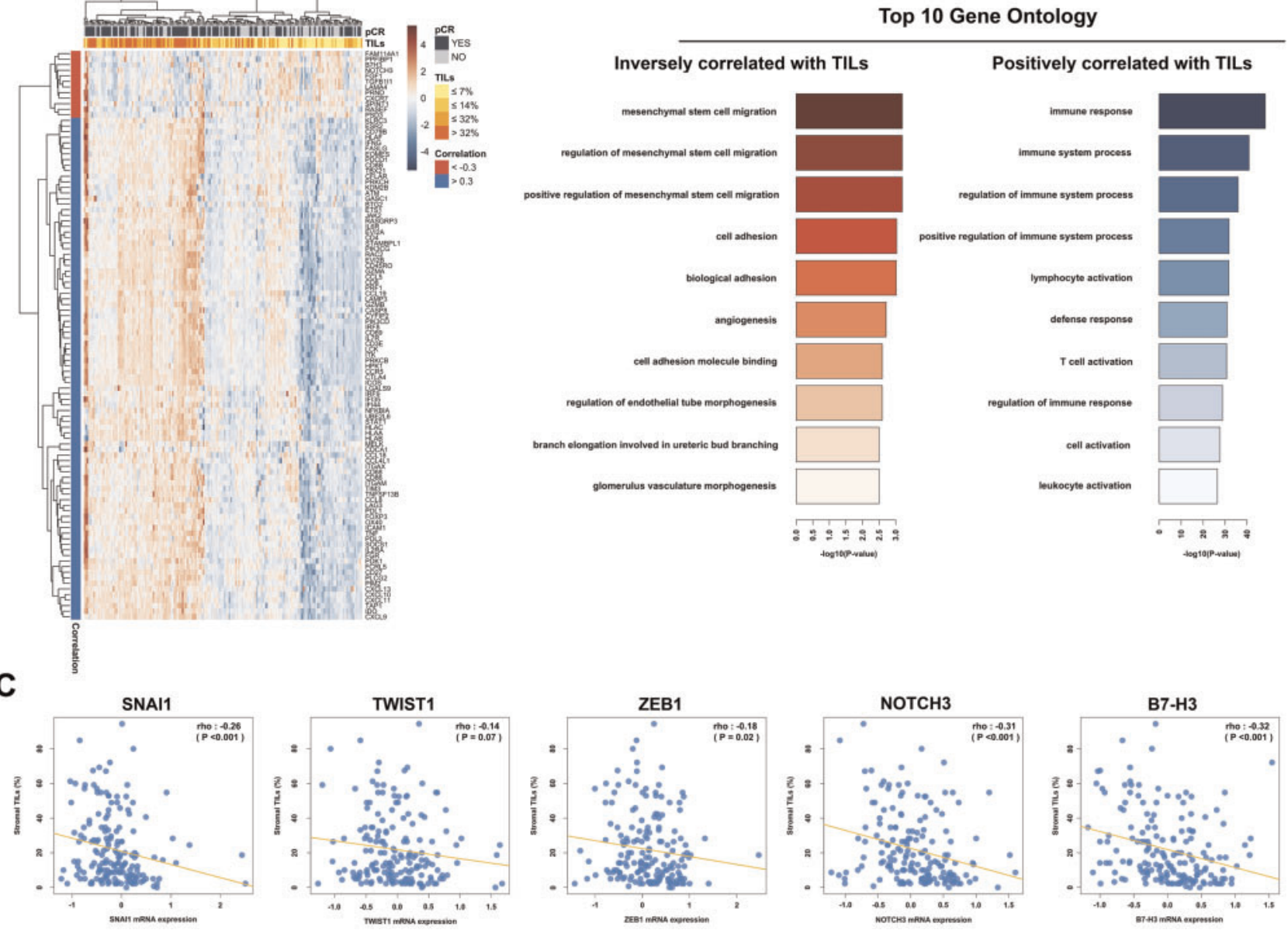

Figure 4. A) Heat map reflecting the hierarchical clustering of the statistically significantly correlated genes with baseline tumor-infiltrating lymphocytes (TILs). Twosided Spearman correlation was used in the analysis, and correction for multitesting (False Discovery Rate [FDR]) was performed. Only genes with $\mid$ rho $\mid \geq .30$ and FDR $<0.05$ were considered statistically significant. B) Gene ontology analysis of the statistically significantly inversely correlated and positively correlated genes with baseline TILs. C) Correlation plot for the epithelial-to-mesenchymal transition (EMT) markers with baseline TIL levels. Two-sided Spearman correlation was used in the analysis. Only genes with $\mathrm{P}<.05$ were considered statistically significant. $\mathrm{pCR}=$ pathological complete response; TIL = tumor-infiltrating lymphocyte.

percentage and correction for multiple testing, increased pCR rates were associated with high expression of immune signatures related to tertiary lymphoid structures and a signature associated with benefit from adjuvant trastuzumab, as well as with high expression of genes associated with CD8+ T-cell cytolytic activity (GZMA, GZMB, PRF1) and with T-helper phenotype 1 (IFNG). Our results extend previously reported associations between PCR and IFNG in patients who received anti-HER2 treatment in the context of the Neosphere trial (5). The fact that immune signatures and single genes were not significantly associated with EFS in our study can be related to the fact that our study is underpowered to detect such associations; thus further studies are needed in this respect.

Furthermore, in order to better understand TIL biology, we evaluated associations between TIL percentage at baseline and the expression of 800 genes related to breast cancer. As expected, increasing levels of TILs were positively correlated with immune response genes. Interestingly, TILs were inversely correlated with the expression of genes associated with EMT, angiogenesis, and T-cell inhibition, such as SNAIL1, ZEB1, NOTCH3, and B7-H3. Our results are in line with preclinical evidence suggesting that snail-induced EMT promotes immunosuppression in melanoma (27) and that mesenchymal stromal cells can suppress the immune response (28).

Our study has some limitations. There were only 64 patients with invasive residual disease and evaluable TILs; thus the study on the association between TILs at surgery and EFS is largely underpowered. Additionally, concerning the associations between baseline TILs and either PCR or EFS, all patients received dual HER2 blockade; thus, we cannot explore single vs dual HER2 blockade biomarker questions. However, this is the first study to explore association of TILs at diagnosis with PCR and EFS for patients with HER2-positive early breast cancer who have received standard preoperative chemotherapy with trastuzumab and pertuzumab. Moreover, we acknowledge that, currently, there is not a validated cutoff for low vs high TIL percentage in this setting. Finally, our gene expression data are hypothesis-generating and need further validation.

In summary, evaluation of baseline TILs might help tailor treatment options for high-risk HER2-positive breast cancer patients who receive neoadjuvant chemotherapy and dual HER2 blockade with trastuzumab and pertuzumab. Further studies are needed to validate our results. 


\begin{tabular}{lcccc} 
Variable & OR & \multicolumn{1}{c}{$\mathbf{C l}$} & p-value & FDR \\
\hline Her2 Enriched (PAM50) & 1.36 & 0.58 to 3.19 & 0.5 & 0.5 \\
\hline ESR1 & 1.14 & 0.4 to 3.35 & 0.8 & 0.8 \\
ERBB2 & 3.48 & 1.37 to 9.38 & 0.01 & 0.03 \\
\hline immune_STAT1 & 3.09 & 0.92 to 11.25 & 0.08 \\
immune_TLS & 5.79 & 1.56 to 24.22 & 0.01 & 0.03 \\
immune_trastuzumab & 4.08 & 1.42 to 12.62 & 0.01 & 0.03 \\
\hline GZMA & 6.21 & 1.77 to 24.48 & 0.006 & 0.03 \\
GZMB & 6.51 & 1.73 to 27.58 & 0.008 & 0.03 \\
PRF1 & 22.29 & 3.75 to 170.44 & 0.001 & 0.02 \\
FOXP3 & 2.14 & 0.57 to 8.98 & 0.3 & 0.3 \\
CTLA4 & 4.08 & 0.91 to 20.63 & 0.07 & 0.1 \\
PDL1 & 2.66 & 0.65 to 12.45 & 0.2 & 0.3 \\
PD1 & 4.46 & 1.09 to 21.52 & 0.05 & 0.09 \\
IL4 & 1.36 & 0.55 to 3.53 & 0.5 & 0.5 \\
IFNG & 5.36 & 1.49 to 22.27 & 0.01 & 0.03 \\
\hline & & & & \\
\hline
\end{tabular}

Figure 5. Adjusted odds ratios (ORs) for pathological complete response for a unit increase in baseline single gene/gene signature score using logistic regression (adjusted for baseline clinicopathological characteristics including age, histology grade, estrogen receptor, clinical stage, chemotherapy, and baseline tumor-infiltrating lymphocytes). Horizontal bars represent the $95 \%$ confidence intervals of the ORs. The Wald test was used to obtain P values and correction for multiple testing using the Benjamini-Hochberg procedure. Characteristics with statistically significant (FDR $<0.05$ ) and non-statistically significant effects are shown in black and gray, respectively. $\mathrm{CI}=$ confidence interval; $\mathrm{FDR}=$ false discovery rate; $\mathrm{OR}=$ odds ratio.

\section{Funding}

CS is supported by the Breast Cancer Research Foundation (BCRF) and the Fonds Nationale de la Recherche Scientifique (FNRS). RS is supported by a grant from the Breast Cancer Research Foundation, New York.

\section{Notes}

Affiliations of authors: Department of Medical Oncology (MI, CS), Breast Cancer Translational Research Laboratory (MI, SR, YB, CD, FR, MM, DV, CS), J. C. Heuson, Institut Jules Bordet, Université Libre de Bruxelles, Brussels, Belgium; Molecular Immunology Unit, Institut Jules Bordet, Université Libre de Bruxelles, Brussels, Belgium (GVdE, KWG); Department of Pathology, GZA, Antwerp, Belgium (SR); University of Milan, Fondazione Istituto di Ricovero e Cura a Carattere Scientifico Istituto Nazionale Tumori, Milan, Italy (MF, EB); Ramon y Cajal University Hospital, Madrid, Spain (EH, JC); Oncology Biomarker Development, Genentech Inc., Basel, Switzerland (VM, AK); Oncology Biomarker Development, Genentech Inc., South San Francisco, CA (HMS, TRW); Vall d'Hebron Institute of Oncology (VHIO), Barcelona, Spain (JC); Divison of Gynecologic Oncology, National Center for Tumor Diseases, University Hospital, Heidelberg, Germany (AS).

The funders had no role in the design of the study; the collection, analysis, or interpretation of the data; the writing of the manuscript; or the decision to submit the manuscript for publication.
MI, RS, and JC were consultants for ROCHE. VM, AK, HS, and TW are employees of and own ROCHE/Genentech stocks. SA received honoraria from ROCHE and Cellgene. MI, CD, and CS received funding from Les Amis de Bordet.

This work was presented in part at the American Society for Clinical Oncology 2016 annual meeting.

\section{References}

1. Denkert C, Loibl S, Noske A, et al. Tumor-associated lymphocytes as an independent predictor of response to neoadjuvant chemotherapy in breast cancer. J Clin Oncol. 2010; 28(1):105-113.

2. Salgado R, Denkert C, Campbell C, et al. Tumor-infiltrating lymphocytes and associations with pathological complete response and event-free survival in HER2-positive early-stage breast cancer treated with lapatinib and trastuzumab: A secondary analysis of the NeoALTTO trial. JAMA Oncol. 2015;1(4): 448-454.

3. Ignatiadis M, Singhal SK, Desmedt C, et al. Gene modules and response to neoadjuvant chemotherapy in breast cancer subtypes: A pooled analysis. J Clin Oncol. 2012;30(16):1996-2004.

4. Fumagalli $D$, Venet $D$, Ignatiadis $M$, et al. RNA sequencing to predict response to neoadjuvant anti-HER2 therapy: A secondary analysis of the NeoALTTO randomized clinical trial. JAMA Oncol. 2016 Sep 29. doi: 10.1001/jamaoncol.2016.3824. [Epub ahead of print].

5. Bianchini G, Pusztai L, Pienkowski T, et al. Immune modulation of pathologic complete response after neoadjuvant HER2-directed therapies in the NeoSphere trial. Ann Oncol. 2015;26(12):2429-2436.

6. Loi S, Michiels S, Salgado R, et al. Tumor infiltrating lymphocytes are prognostic in triple negative breast cancer and predictive for trastuzumab benefit in early breast cancer: Results from the FinHER trial. Ann Oncol. 2014;25(8): 1544-1550.

7. Perez EA, Ballman KV, Tenner KS, et al. Association of stromal tumorinfiltrating lymphocytes with recurrence-free survival in the N9831 adjuvant trial in patients with early-stage HER2-positive breast cancer. JAMA Oncol. 2016;2(1):56-64.

8. Schneeweiss A, Chia S, Hickish T, et al. Pertuzumab plus trastuzumab in combination with standard neoadjuvant anthracycline-containing and 
anthracycline-free chemotherapy regimens in patients with HER2-positive early breast cancer: A randomized phase II cardiac safety study (TRYPHAENA). Ann Oncol. 2013;24(9):2278-2284.

9. Buisseret L, Desmedt C, Garaud S, et al. Reliability of tumor-infiltrating lymphocyte and tertiary lymphoid structure assessment in human breast cancer. Mod Pathol. 2017;30(9):1204-1212.

10. Salgado R, Denkert C, Demaria S, et al. The evaluation of tumor-infiltrating lymphocytes (TILs) in breast cancer: Recommendations by an International TILs Working Group 2014. Ann Oncol. 2015;26(2):259-271.

11. Dieci MV, Radosevic-Robin N, Fineberg S, et al. Update on tumor-infiltrating lymphocytes (TILs) in breast cancer, including recommendations to assess TILs in residual disease after neoadjuvant therapy and in carcinoma in situ: A report of the International Immuno-Oncology Biomarker Working Group on Breast Cancer. Semin Cancer Biol. 2017 Oct 9. pii: S1044-579X(17)30217-1. doi: 10.1016/j.semcancer.2017.10.003. [Epub ahead of print]

12. Wilson TR, Yu J, Lu X, et al. The molecular landscape of high-risk early breast cancer: Comprehensive biomarker analysis of a phase III adjuvant population. NPJ Breast Cancer. 2016;2:16022.

13. Carey LA, Berry DA, Cirrincione CT, et al. Molecular heterogeneity and response to neoadjuvant human epidermal growth factor receptor 2 targeting in CALGB 40601, a randomized phase III trial of paclitaxel plus trastuzumab with or without lapatinib. J Clin Oncol. 2016;20;34(6):542-549.

14. Desmedt C, Haibe-Kains B, Wirapati P, et al. Biological processes associated with breast cancer clinical outcome depend on the molecular subtypes. Clin Cancer Res. 2008;14(16):5158-5165.

15. Gu-Trantien C, Loi S, Garaud S, et al. CD4(+) follicular helper T cell infiltration predicts breast cancer survival. J Clin Invest. 2013;123(7):2873-2892.

16. Perez EA, Thompson EA, Ballman KV, et al. Genomic analysis reveals that immune function genes are strongly linked to clinical outcome in the North Central Cancer Treatment Group n9831 Adjuvant Trastuzumab Trial. J Clin Oncol. 2015;33(7):701-708.

17. McShane LM, Altman DG, Sauerbrei W, Taube SE, Gion M, Clark GM. Reporting recommendations for tumor marker prognostic studies. J Clin Oncol. 2005;23(36):9067-9072.
18. FDA. 2013. https://www.cancer.gov/about-cancer/treatment/drugs/fdapertuzumab\#Anchor-NeoHER2. Accessed April 26, 2018

19. von Minckwitz G, Procter M, de Azambuja E, et al. Adjuvant pertuzumab and trastuzumab in early HER2-positive breast cancer. N Engl J Med. 2017;377(2): 122-131.

20. Dieci MV, Criscitiello C, Goubar A, et al. Prognostic value of tumor-infiltrating lymphocytes on residual disease after primary chemotherapy for triplenegative breast cancer: A retrospective multicenter study. Ann Oncol. 2014; 25(3):611-618.

21. Loi S, Adams S, Schmid P, et al. Relationship between tumor infiltrating lymphocyte levels and response to pembrolizumab in metastatic triple negative breast cancer: Results from Keynote-086. Ann Oncol. 2017; 28(supp 5).

22. Rizvi NA, Hellmann MD, Snyder A, et al. Cancer immunology. Mutational landscape determines sensitivity to PD-1 blockade in non-small cell lung cancer. Science. 2015;348(6230):124-128.

23. Snyder A, Makarov V, Merghoub T, et al. Genetic basis for clinical response to CTLA-4 blockade in melanoma. N Engl J Med. 2014;371(23): 2189-2199.

24. McGranahan N, Furness AJ, Rosenthal R, et al. Clonal neoantigens elicit T cell immunoreactivity and sensitivity to immune checkpoint blockade. Science. 2016;351(6280):1463-1469.

25. Criscitiello C, Esposito A, Gelao L, et al. Immune approaches to the treatment of breast cancer, around the corner? Breast Cancer Res. 2014;16(1): 204.

26. Savas P, Salgado R, Denkert C, et al. Clinical relevance of host immunity in breast cancer: From TILs to the clinic. Nat Rev Clin Oncol. 2016; 13(4): 228-241.

27. Kudo-Saito C, Shirako H, Takeuchi T, Kawakami Y. Cancer metastasis is accelerated through immunosuppression during Snail-induced EMT of cancer cells. Cancer Cell. 2009;15(3):195-206.

28. Poggi A, Giuliani M. Mesenchymal stromal cells can regulate the immune response in the tumor microenvironment. Vaccines (Basel). 2016;(4). 